\title{
Controlling the core-to-core distance of vortex pairs in exchange-biased iron elliptical nanoelements
}

Felipe F. Oliveira, Thiago R. S. Moura, A. S. Carriço, Ana L. Dantas, and G. O. G. Rebouças

Citation: Journal of Applied Physics 111, $07 D 102$ (2012);

View online: https://doi.org/10.1063/1.3670978

View Table of Contents: http://aip.scitation.org/toc/jap/111/7

Published by the American Institute of Physics

\section{Articles you may be interested in}

Tailoring the vortex core in confined magnetic nanostructures

Journal of Applied Physics 111, 07D116 (2012); 10.1063/1.3675989

Nucleation of vortex pairs in exchange biased nanoelements

Journal of Applied Physics 109, 07D314 (2011); 10.1063/1.3537924

Controlling the vortex core of thin Permalloy nano-cylinders dipolar coupled to Co polarizers

Journal of Applied Physics 115, 17D110 (2014); 10.1063/1.4860056

Thermal hysteresis of interface biased dipolar coupled nanoelements

Journal of Applied Physics 113, 17D710 (2013); 10.1063/1.4795868

Thermal hysteresis of interface biased ferromagnetic dots

Journal of Applied Physics 102, 123907 (2007); 10.1063/1.2827478

Dipolar field effects on the critical current for spin transfer switch of iron and permalloy nanoelements Journal of Applied Physics 115, 17D130 (2014); 10.1063/1.4865317

\section{Scilight}

Sharp, quick summaries illuminating the latest physics research

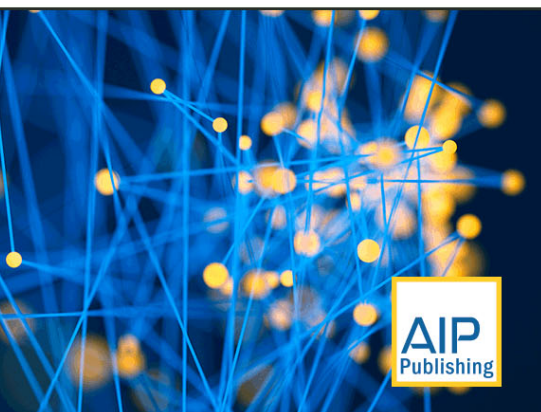




\title{
Controlling the core-to-core distance of vortex pairs in exchange-biased iron elliptical nanoelements
}

\author{
Felipe F. Oliveira, ${ }^{1}$ Thiago R. S. Moura, ${ }^{1}$ A. S. Carriço, ${ }^{1, a)}$ Ana L. Dantas, ${ }^{2}$ \\ and G. O. G. Rebouças ${ }^{3}$ \\ ${ }^{1}$ Departamento de Física, Universidade Federal do Rio Grande do Norte, Natal, RN 59072-970, Brasil \\ ${ }^{2}$ Departamento de Física, Universidade do Estado do Rio Grande do Norte, Mossoró, RN 59610-210, Brasil \\ ${ }^{3}$ Departamento de Física, Universidade Federal do Semi-Arido, Angicos, RN 59515-000, Brasil
}

(Presented 3 November 2011; received 23 September 2011; accepted 14 October 2011; published online 10 February 2012)

\begin{abstract}
We report a theoretical study of vortex pairs in exchange-biased elliptical iron nanoelements. We show that the remanent state may be tailored to fit vortex pairs with opposite chiralities separated by a diamond-like domain. Flat nanoelements with lateral dimensions ranging from $115 \mathrm{~nm} \times 425 \mathrm{~nm}$ to $195 \mathrm{~nm} \times 425 \mathrm{~nm}$ have the core-to-core distance tunable by the interface field strength. (C) 2012 American Institute of Physics. [doi:10.1063/1.3670978]
\end{abstract}

The remanent state of elliptical nanoelements can be designed to fit a vortex pair along the major axis by tailoring the nanoelement aspect ratio and controlling the nucleation mechanism. Vortex pairs with opposite or with the same chiralities have been reported. ${ }^{1-5}$ Controlling the distance between vortex cores may be attractive for the purpose of tailoring the vortex core size and polarity, as well as the vortex pair excitations. ${ }^{6-8}$

We show presently that one may control the vortex pair structure of soft nanoelements by exchange bias.

Nano-patterning soft ferromagnetic materials leads to an enhancement of demagnetizing field effects. ${ }^{9}$ Under appropriate size limits, the competition between the dipolar and the intrinsic exchange fields may favor the formation of flux closure states, such as single vortex and double vortex states. ${ }^{3}$

Interface exchange coupling to a large uniaxial anisotropy antiferromagnetic substrate adds an extra means of tailoring the magnetic pattern. Larger areas with interface spins parallel to the interface field are favored. Thus, there are good chances of breaking the balance between dipolar and intrinsic exchange energies and changing the magnetic structure of a vortex pair.

In this paper, we report a theoretical study of vortex pairs in exchange-biased elliptical iron nanoelements. We consider flat iron nanoelements, with height of the order of the iron exchange length, and lateral dimensions ranging from $115 \mathrm{~nm} \times 425 \mathrm{~nm}$ to $195 \mathrm{~nm} \times 425 \mathrm{~nm}$.

Choosing flat nanoelements restricts the possible magnetic states. Only magnetic states uniform along the normal to the surface are allowed. Thus, one may focus on effects of the lateral dimensions.

We show that there is a large impact of the nucleation process and the strength of the interface field in the magnetic pattern of vortex pairs. Furthermore, the modifications induced by interface bias are larger for narrow nanoelements.

The core-to-core distance may be tuned to vary from $50 \%$ up to twice the unbiased value for the Fe $115 \mathrm{~nm}$ $\times 425 \mathrm{~nm} \times 20 \mathrm{~nm}$ nanoelement. For $\mathrm{Fe} 195 \mathrm{~nm} \times 425 \mathrm{mn}$

\footnotetext{
${ }^{\text {a)} E l e c t r o n i c ~ m a i l: ~ a s c a r r i c o @ g m a i l . c o m . ~}$
}

$\times 20 \mathrm{~nm}$, the core-to-core distance varies from $56 \%$ up to $175 \%$ of the unbiased value. As shown in Fig. 1, this corresponds to maximum compression and stretching of the coreto-core distance for these nanoelements.

The uniaxial anisotropy easy axis and the interface field are both in-plane and along the minor axis ( $x$ axis), and the antiferromagnetic substrate is held frozen in the antiferromagnetic order. The major axis is along the y-axis.

We use cubic simulation cells with edge $d=5 \mathrm{~nm}$, and the energy density is given by

$$
\begin{aligned}
E= & \frac{A}{d^{2}} \sum_{j} \sum_{k}\left(1-\hat{m}_{j} \cdot \hat{m}_{k}\right)-M_{S} \vec{H}_{i n t} \cdot \sum_{i} \hat{m}_{i} \\
& -M_{S} \vec{H} \cdot \sum_{j} \hat{m}_{j}-K \sum_{j}\left(m_{j}^{x}\right)^{2} \\
& +\frac{M_{S}^{2}}{2} \sum_{j} \sum_{k}\left(\frac{\hat{m}_{j} \cdot \hat{m}_{k}}{n_{j k}^{3}}-\frac{3\left(\hat{m}_{j} \cdot \hat{n}_{j k}\right)\left(\hat{m}_{k} \cdot \hat{n}_{j k}\right)}{n_{j k}^{5}}\right),
\end{aligned}
$$

where the first term is the intrinsic exchange energy of nearest-neighbor cells and $A$ is the exchange stiffness. The second term is the interface exchange energy, restricted to the interface cells. The third and fourth terms are the Zeeman and anisotropy energies, and the last term is the magnetostatic energy. $M_{S}$ is the saturation magnetization, $\hat{m}_{i}$ is the direction of the magnetic moment of the $i-t h$ cell, and $n_{i j}$ is the distance between the cells $i$ and $j$ in units of cell size $d$.

For the iron magnetic parameters, we use $M_{S}=1.7 \times 10^{6}$ $\mathrm{A} / \mathrm{m}, A=2.5 \times 10^{-11} \mathrm{~J} / \mathrm{m}$ and $K=4.7 \times 10^{-4} \mathrm{~J} / \mathrm{m}^{3}$. The effective exchange field, coupling neighboring cells, is $H_{E}=A / M_{S} d^{2}$.

For each value of the external field strength, the equilibrium configuration is found by seeking a set of directions of the moments in all cells $\left(\hat{m}_{i}, i=1, \ldots, N\right)$, which makes the torque smaller than $10^{-26} \mathrm{~J}$ in any one of the cells. ${ }^{3,9}$

We have found that unbiased nanoelements display a double vortex pattern consisting of vortex cores with opposite chirality separated by a diamond-like domain (see panels in Fig. 2). The vortex-domain-vortex (VDV) pattern is stable for positive and negative values of the interface exchange field. 


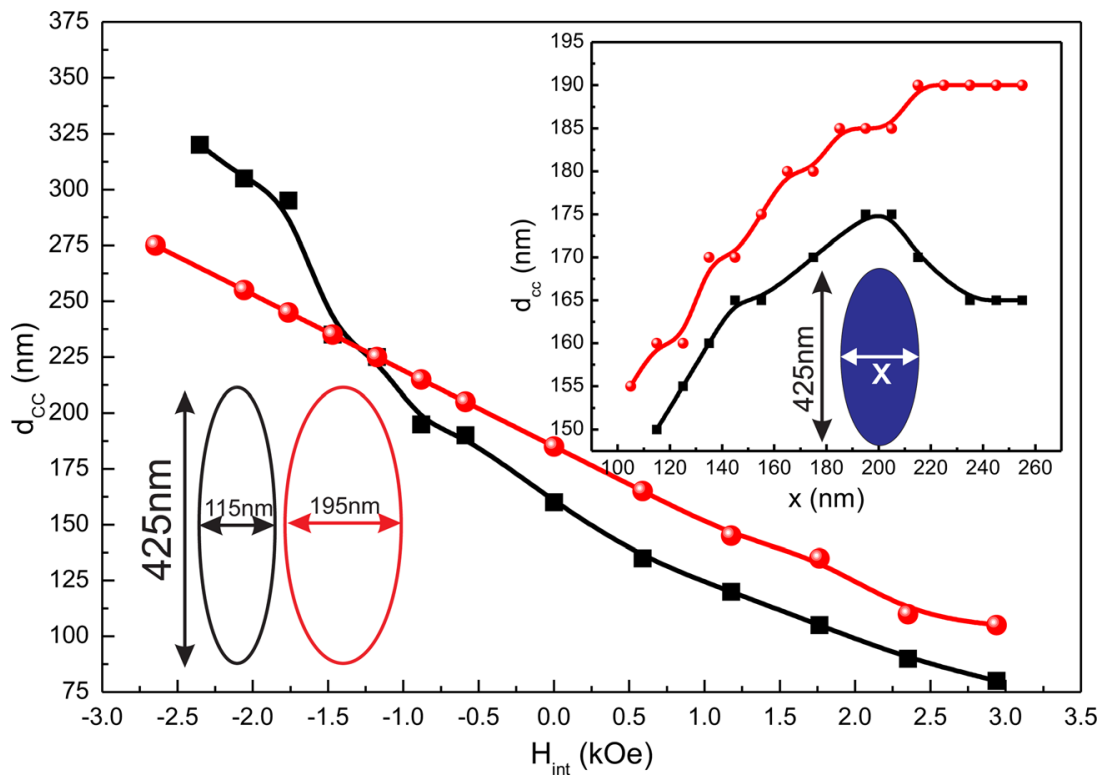

FIG. 1. (Color online) Interface field effect on the coreto-core distance of Fe $115 \mathrm{~nm} \times 425 \mathrm{~nm} \times 20 \mathrm{~nm}$ (square symbol curve) and Fe $195 \mathrm{~nm} \times 425 \mathrm{~nm} \times 20 \mathrm{~nm}$ (circle symbol curve). In the inset, we show the core-to-core distance of unbiased Fe $x(n m) \times 425 \mathrm{~nm}$, with height of $15 \mathrm{~nm}$ (square symbol curve) and $20 \mathrm{~nm}$ (circle symbol curve).

We start from saturation at large values of the external field (6 kOe suffices for all nanoelements in this study), and the equilibrium pattern for a given value of the external field is used as the initialization pattern for the next value of the external field. The remanent state is found by starting from saturation with the external field either parallel or opposite to the interface field direction. Each case corresponds to a nucleation process, leading to either decreasing or increasing the core-to-core distance, as compared with the unbiased value.

As shown in Fig. 2 for an unbiased Fe $195 \mathrm{~nm} \times 425 \mathrm{~nm}$ $\times 20 \mathrm{~nm}$, the nucleation of a non-uniform phase occurs at an external field of $0.7 \mathrm{kOe}$ and corresponds to a curling state. ${ }^{4}$ At point $\mathrm{A}$, for an external field value of $0.4 \mathrm{kOe}$, a VDV state is formed with most of the magnetic moments along the external field direction. The domain between the vortex cores has the magnetization aligned opposite to the external field, and the core-to-core distance is $115 \mathrm{~nm}$ (see panel A in Fig. 2).
By further reducing the external field and the Zeeman energy, the central domain and the core-to-core distance get larger, reaching a core-to-core distance of $185 \mathrm{~nm}$ at remanence $(\mathrm{H}=0)$.

For an interface field pointing opposite (parallel) to the external field, the separation between vortex cores gets larger (smaller) at remanence. Panel C of Fig. 2 shows the vortex pair for an interface field of $1.7 \mathrm{kOe}$ with a core-to-core distance of $110 \mathrm{~nm}$ ( $60 \%$ of the unbiased value $-185 \mathrm{~nm}$ ).

The vortex pair pattern of narrow nanoelements is likely to suffer a larger impact of interface bias. The interface field produces modifications of the balance between dipolar and intrinsic energies, favoring larger areas with spins aligned with the interface field direction. This requires larger changes of the core-to-core distance in the case of narrow nanoelements.

The unbiased Fe $195 \mathrm{~nm} \times 425 \mathrm{~nm} \times 20 \mathrm{~nm}$ nanoelement displays a vortex pair with core-to-core distance of $185 \mathrm{~nm}$,
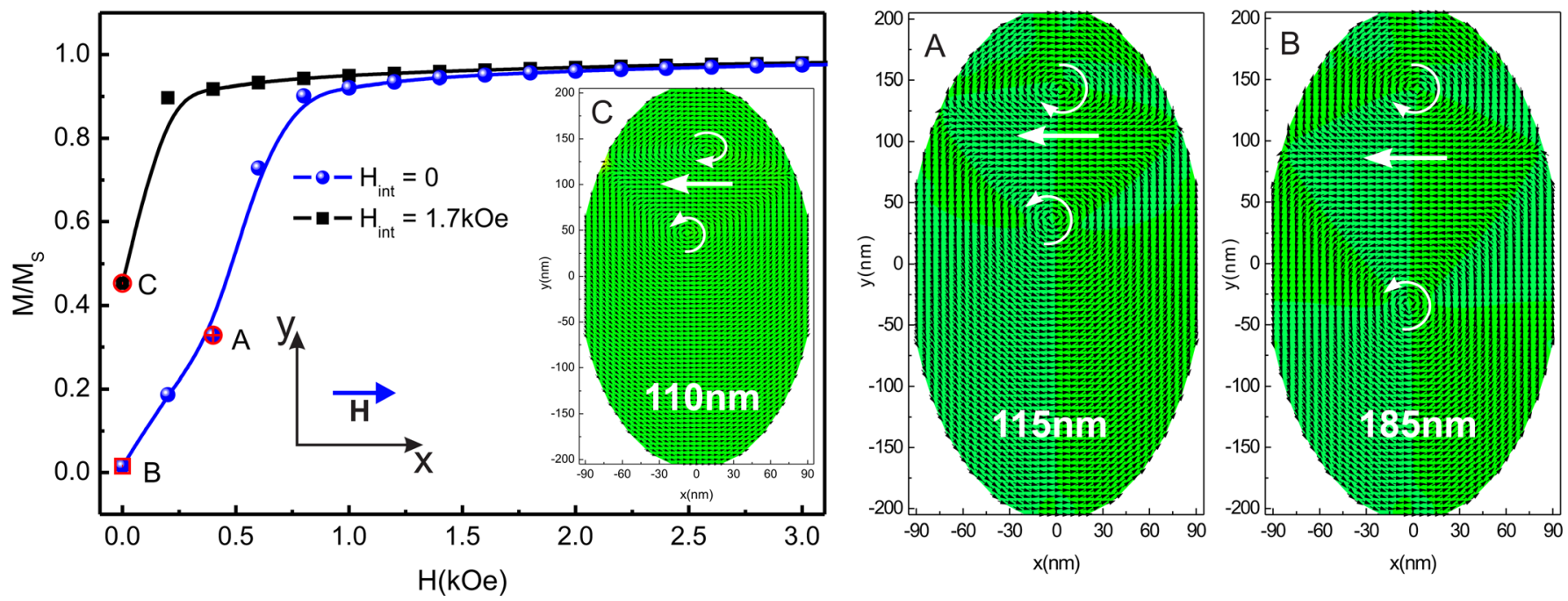

FIG. 2. (Color online) Magnetization of an unbiased (circle symbol curve) and interface biased (square symbol curve) $195 \mathrm{~nm} \times 425 \mathrm{~nm} \times 20 \mathrm{~nm}$ iron nanoelement. The panels show the magnetic structure at the vortex nucleation field (A) and at remanence (B) for the unbiased nanoelement. Panel C shows the magnetic pattern of the interface-biased nanoelement for an interface field strength of $1.7 \mathrm{kOe}$. The number in each panel is the value of the core-to-core distance. 

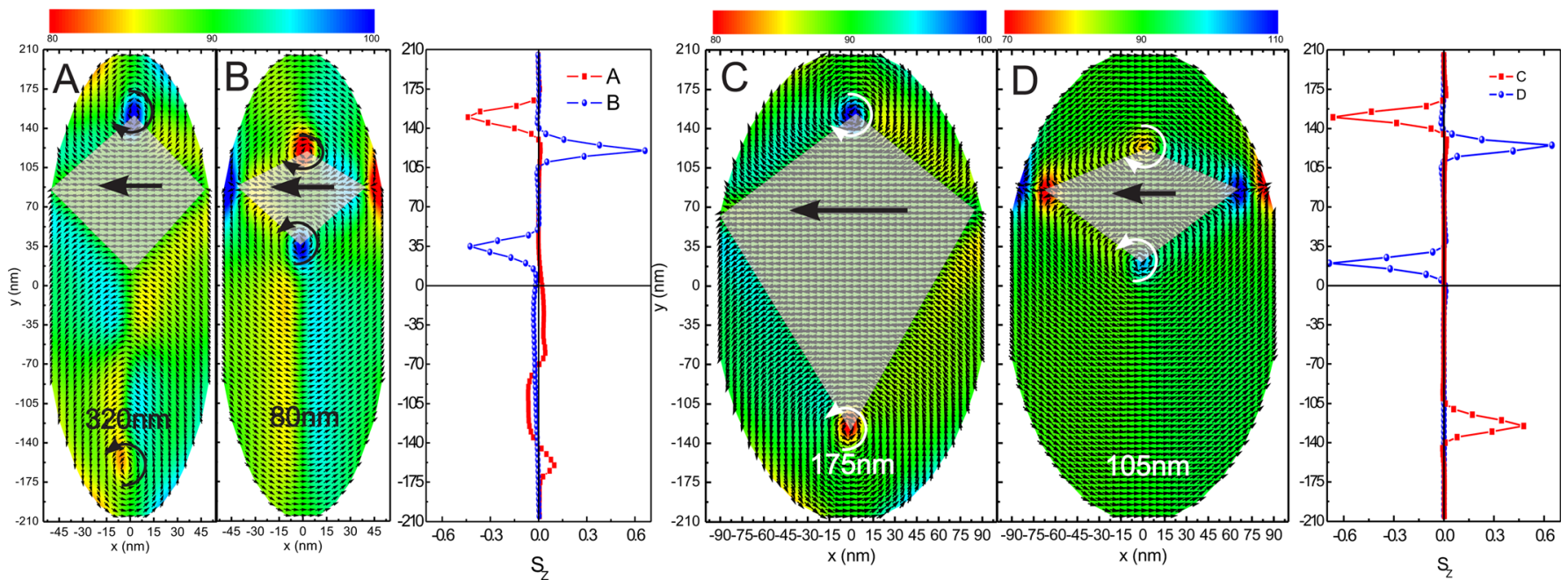

FIG. 3. (Color online) Magnetic phases for maximum stretching and compression of the core-to-core distance of the Fe $115 \mathrm{~nm} \times 425 \mathrm{~nm} \times 20 \mathrm{~nm}$ nanoelement (panels A and B) and the Fe $195 \mathrm{~nm} \times 425 \mathrm{~nm} \times 20 \mathrm{~nm}$ nanoelement (panels C and D). We also show the z-component of the magnetization along the $\mathrm{y}$-axis. The number in each panel is the value of the core-to-core distance, and the color bar code indicates the values of the angle with the $\mathrm{z}$-axis.

which is around $45 \%$ of the major axis length. Exchange bias produces a large variation of the core-to-core distance, ranging from $105 \mathrm{~nm}$ for $H_{\text {int }}=2.9 \mathrm{kOe}$, up to $275 \mathrm{~nm}(65 \%$ of the major axis length) for $H_{\text {int }}=-2.64 \mathrm{kOe}$ (opposite to the external field).

Exchange bias produces larger effects in the $\mathrm{Fe}$ $115 \mathrm{~nm} \times 425 \mathrm{~nm} \times 20 \mathrm{~nm}$ nanoelement. The core-to-core distance for the unbiased nanoelement is $160 \mathrm{~nm}$ (around $38 \%$ of the major axis length). In this case, the overall change in the core-to-core distance is from $80 \mathrm{~nm}$ up to $320 \mathrm{~nm}$.

In Fig. 3, we also show the polarity of vortex pair cores. As seen in the plots of $S_{Z}$, the polarity of vortex cores of Fe $195 \mathrm{~nm} \times 425 \mathrm{~nm} \times 20 \mathrm{~nm}$ does not vary much with compression or stretching of the core-to-core distance. On the other hand, there is a large variation of $S_{Z}$ from core to core for the Fe $115 \mathrm{~nm} \times 425 \mathrm{~nm} \times 20 \mathrm{~nm}$ nanoelement. When the cores are compressed together, the values of $S_{Z}$ are almost the same for both cores. However, for large stretching, there is a large asymmetry: one of the vortex cores has a negligible $S_{Z}$-component and is located at the far end of the nanoelement, away from the diamond domain corner.

In summary, we have shown that the magnetic structure of vortex pairs of Fe nanoelements can be largely influenced by interface exchange biasing. The core-to-core distance may be largely tuned by the interface field in narrow nanoelements.
In the Fe $115 \mathrm{~nm} \times 425 \mathrm{~nm} \times 20 \mathrm{~nm}$, the core-to-core distance may be varied from $19 \%$ and $75 \%$ of the major axis. This also applies to thinner nanoelements $(\mathrm{h}=15 \mathrm{~nm})$, and the inset in Fig. 1 may indicate the range of core-to-core distances one may achieve by interface bias.

We note that the control of the distance between the cores of vortex pairs may be a key issue for the synchronization of vortex pair dynamics. Interface bias may thus be helpful in designing the spectra of tunnel junction nano-oscillators.

The authors acknowledge financial support from CNPq, CAPES, and FAPERN. The work of A.S.C. was supported by CNPq Grant No. 350773 and the work of A.L.D. was supported by CNPq Grant No. 309676.

${ }^{1}$ T. Okunoa, K. Mibu, and T. Shinjo, J. Appl. Phys. 95, 3612 (2004).

${ }^{2}$ K. S. Buchanan et al., Nature Phys. 1, 172 (2005).

${ }^{3}$ A. S. M. Silva, A. L. Dantas, G. O. G. Rebouças, and A. S. Carriço, J. Appl. Phys. 109, 07D314 (2011).

${ }^{4}$ N. A. Usov, C. R. Chang, and Z. H. Wei, Phys. Rev. B 66, 184431 (2002).

${ }^{5}$ P. Vavassori, et al., Phys. Rev. B 69, 214404 (2004).

${ }^{6}$ F. B. Mancoff, N. D. Rizzo, B. N. Engel, and S. Tehrani, Nature Lett. 437, 393 (2005).

${ }^{7}$ S. Kaka et al., Nature Lett. 437, 389 (2005).

${ }^{8}$ M. van Kampen, L. Lagae, G. Hrkac, T. Schrefl, J.-V. Kim, T. Devolder, and C. Chappert, J. Phys. D: Appl. Phys. 42, 245001 (2009).

${ }^{9}$ G. O. G. Rebouças, A. S. W. T. Silva, A. L. Dantas, R. E. Camley, and A. S. Carriço, Phys. Rev. B 79, 104402 (2009). 